Review Article

\title{
Single Cell Manipulation Technology
}

\author{
Shujing Lin, Di Chen ${ }^{\prime}$, Yao Xie, Xiao Zhi, Qian Wei, Fei Pan, Daxiang Cui \\ Key Lab. for Thin Film and Microfabrication Technology of Ministry of Education, Department of Instrument Science and \\ Engineering, School of Electronic Information and Electrical Engineering, Shanghai Jiao Tong University, Shanghai 200240, China \\ Corresponding author: E-mail: dchen@sjtu.edu.cn
}

Received: Jul. 2, 2015; Accepted: Jul. 20, 2015; Published: Jul. 24, 2015.

Citation: Shujing Lin, Di Chen, Yao Xie, Binbin Lin, Qian Wei, Fei Pan and Daxiang Single Cell Manipulation Technology. Nano Biomed. Eng. 20I5, 7(3), 75-91.

DOI: 10.5101/nbe.v7i3.p75-91.

\begin{abstract}
With microfluidic technique emerging, cell manipulation technology combines with microfluidic become a promising tool for single-cell-level manipulation. To obtain a kind of or single pure target cell for eliminating interference of useless cells in the trial of molecular biology, genetic analysis, proteomics and single cell analysis, various cell manipulation techniques have been developed for recovery specific cells. In this review, we introduce the principles of each cell manipulation technology and overlook the latest achievements of cell manipulation technique by categorizing externally applied manipulation forces: optical, electrical, magnetic, acoustic, mechanical. We also summarize the advantages and drawbacks of each cell manipulation technique.
\end{abstract}

Keywords: Single cell manipulation; Cell trapping; Cell sorting; Cell separation

\section{Introduction}

Since single cell sizes are in a range from several micrometers to hundreds of micrometers were discovered, researchers began to observe and study them. From the mid-1900s, scientists and engineers started to have strong interest in manipulation biological particles techniques, not just in observation of stationary microscopic particles [1]. In order to meet the demands of observation and analysis of cellular and intracellular components, much research have been done to find an effectively way to manipulate cells at single-cell-level by researchers [2-6].

With the microfluidic technology emerging in 1980 s, scientists have devoted a lot of work to improve the performance of microfluidic. In the early days, microfluidic approaches are used in various research fields such as molecular biology, genetic analysis and proteomics, but not cell biology [7]. Due to the advantages of microfluidic, such as precisely control the cellular environment and easy to analyze cellular at the single-cell-level, it is very appropriate for cell biology. Hence, microfluidic technology was introduced into the field of cell biology by researchers step by step. After decades of development, microfluidic technology has been successfully used in the area of manipulation fluid droplets, particles, single cell, and so on [8].

Nowadays, microfluidic technique plays a critical role in cell manipulation field. Cell manipulation technology, which is applied to sort cells, separate cells, trap cells, isolate cells, move cells, recover cells and so on [9-14], combined with microfluidic technique make the ways of manipulation cells at 
single-cell-level more precision and convenient than ever before. According to different cell manipulation forces generation principles, cell manipulation technology can be divided into optical manipulation technique, magnetic manipulation technique, electrical manipulation technique, acoustic manipulation technique and mechanical manipulation technique. This review will introduce the principles of each cell manipulation technique, and overview the current situation of them, and then show the advantages and drawbacks of each cell manipulation technique at the end.

\section{Single cell manipulation technology \\ Optical manipulation technique}

A concept of an optical trapping technique was first reported by Ashkin at Bell Labs in 1970, they have demonstrated that micro-sized particles had been accelerated and trapped in stable optical potential wells using only the force of radiation pressure from a continuous laser [15]. Shortly afterward, optical trapping and manipulation of viruses and bacteria by laser radiation pressure were demonstrated with singlebeam gradient traps by Ashkin and his colleagues [16]. From optical trapping, also known as "optical tweezers", was first reported to now, there have been many improvements of cell optical manipulation.

Optical manipulation technique has found applications in both physics and biology. However, how to use a laser beam to manipulate particles or bioparticles? An optical manipulation is formed by tightly focusing a laser beam with an objective lens of high numerical aperture (NA). A dielectric particle near or in the focus will experience a force due to a portion of the scattering of incident photons momentum of will transfer to the particle. This optical force has traditionally been decomposed into two components: scattering force and gradient force [17]. The scattering force can be imagined photons as fire horses pushing the particle moving towards of the light propagation direction and the effective scattering force can be calculated. The scattering force of a sphere is

$$
\begin{aligned}
& F_{\text {scatt }}=\frac{I_{0} \sigma n_{m}}{c} \\
& \sigma=\frac{128 \pi^{5} a^{6}}{3 \lambda^{4}}\left(\frac{m^{2}-1}{m^{2}+2}\right)^{2}
\end{aligned}
$$

where $I_{0}$ is the intensity of the incident light, $\sigma$ is the scattering cross section of the sphere, $n_{m}$ and $n_{p}$ is the index of refraction of the medium and particle respective, $c$ is the speed of light in vacuum, $a$ is the radius of sphere, $m$ is the ratio of the index of refraction of the particle to the index of the medium $\left(n_{p} / n_{m}\right)$, and $\lambda$ is the wavelength. The gradient force arises from the interaction of the induced dipole with an inhomogeneous field. The gradient force is proportional to the intensity gradient $\left(\nabla I_{0}\right)$, shown in Eq. (3).

$$
F_{\text {grad }}=\frac{2 \pi}{c} a^{3}\left(\frac{m^{2}-1}{m^{2}+2}\right) \nabla I_{0}
$$

Although the theory of optical manipulation is still being developed, the basic principles are still the same. For stable trapping requires the gradient force to dominate, and is achieved when the beam diverges rapidly enough away from the focal point. Intensity gradients in the converging beam pull small particle toward the focus, whereas the radiation pressure of the beam tends to blow them down the optical axis. Under the gradient force dominates condition, a particle can be trapped in three dimensions, near the focal point, shown in Fig. 1 [18].

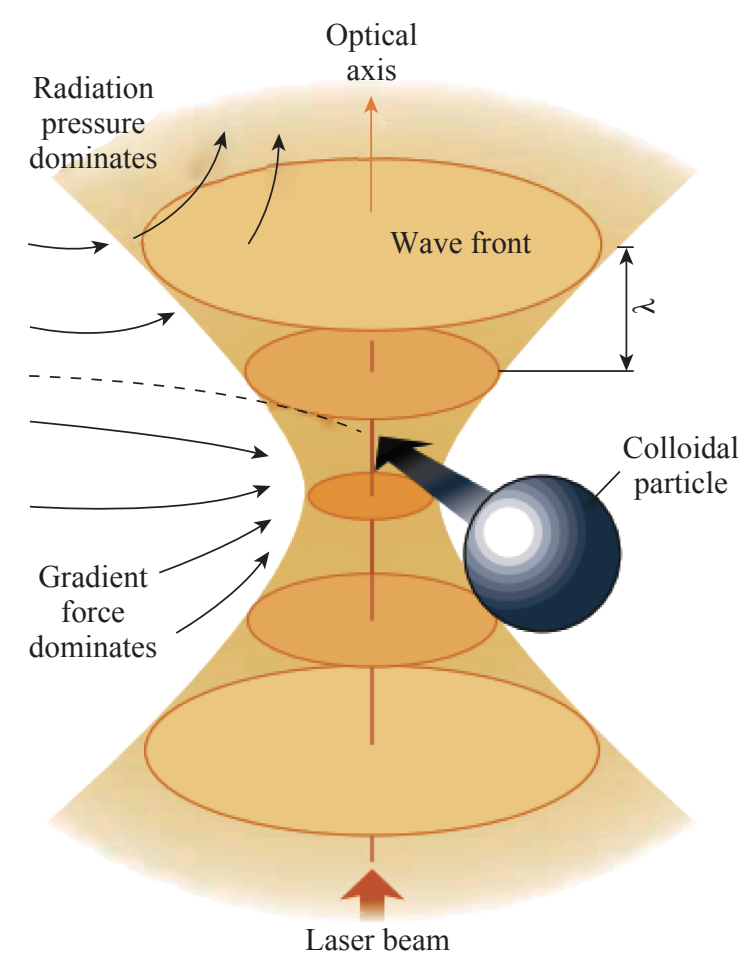

Fig. 1 A diagram of optical force for particle trapping. A dielectric particle will experience a gradient force that pull it toward the focus and a radiation pressure that push it away from the focus, while these two forces are balance, the particle is trapped [18]. 
Based on this principle, researchers developed a kind of tool, which is called optical tweezers, to manipulate particles and bio-particles [19-22]. These particles and bio-particles include cells, bacteria, virus, microbeads, and so on. However, the usefulness of this technique has been limited, particularly in manipulating bio-particles, due to the manipulation laser-beam may cause some potential damage to the bio-samples. In order to characterize photodamage of optical manipulation technique, Neuman characterized photodamage throughout the near infrared region for optical trapping by using Escherichia coli as specimens [23]. They found that the action spectrum for photodamage minima at 830 and $970 \mathrm{~nm}$, and maxima at 870 and $930 \mathrm{~nm}$. A few years later, in order to quantify potentially harmful or misleading heating effects of this technique in biophysical experiments, Peterman demonstrated that the heating effect in water have non-negligible effects in typical biophysical experimental circumstances and it should be taken into account when laser powers of more than $100 \mathrm{~mW}$ are used [24].

Although optical manipulation technique has its limitation in the field of bio-particle manipulation, this technique is still a powerful tool to manipulate bio-particles due to its own advantages, such as highthroughput and high-accuracy. Eriksson and Enger created an environmental gradient between two media in a microfluidic system and used optical tweezers to move a single trapped cell repeatedly between the different environments for analyzing rapid and reversible cytological alterations in single cells, the schematic of the setup is shown in Fig. 2 [25]. The trapped object can be moved within the microfluidic system using the motorized microscope stage to change the position of the channel system relative to the fixed trap.

In particle sorting field, MacDonald demonstrated an optical sorter for microscopic particles that exploits the interaction of particles-biological or otherwise-with an extended, interlinked, dynamically reconfigurable, three-dimensional optical lattice. This concept of optical fractionation can both sort by size and refractive index. The sorting efficiency of this method was approxmatey $100 \%$ [26]. After that, researchers have designed various structures of microfluidics combined with optical manipulation technique for sorting, trapping and separating cells or particles (see Fig. 3). Kovac and Voldman designed a microfluidic chip contains a microwell array that can be passively

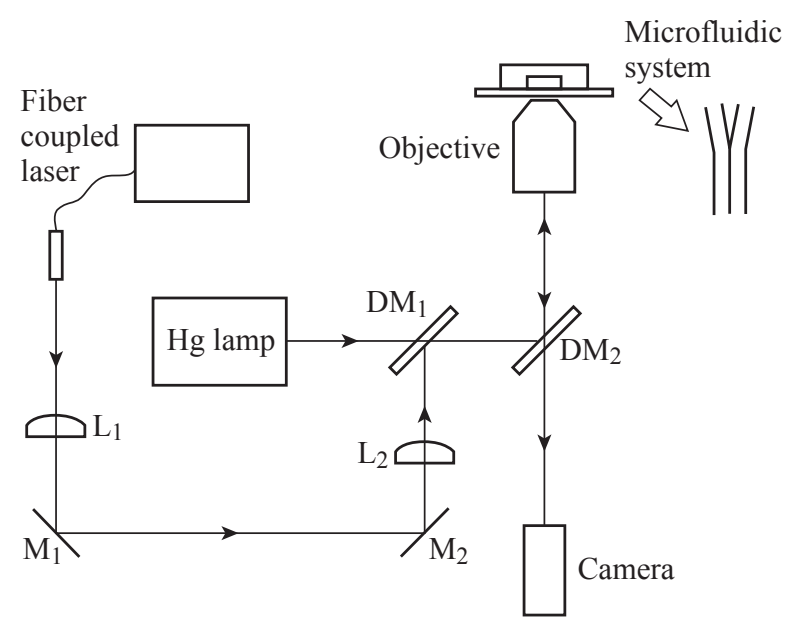

Fig. 2 A schematic of experimental setup of the optical tweezers. In this design, the laser beam through an optical system for manipulation target cells, which are selected by a digital microscope system. This experimental optical tweezers can move the target cells from one side to another side in the microfluidic [25].

loaded with mammalian cells via sedimentation shown in Fig. 3(a). Subsequently, target cells were selected by microscopy, and then, used the scattering force from a focused infrared laser to levitate target cells from their wells into a flow field for collection. This work achieved post-sort purities up to $89 \%$, and up to 155 -fold enrichment of target cells [27]. Wang and his workmates designed a microfluidic chip based on dynamic fluid and dynamic light pattern and a recognition capability of multiple cell features shown in Fig. 3(b). The experiments of sorting yeast cells and human embryonic stem cells are demonstrated by this approach [28]. Kim designed a generic single cell manipulation tool that integrates optical tweezers and microfluidic chip technologies for handling small cell population sorting with high accuracy shown in Fig. 3(c) [29].

Over the last few years, although, optical manipulation technique combine with microfluidic has made a big advancement and been used for clinical applications. There are some issues and drawbacks, such as photodamage, still need to improve and solve.

\section{Electrical manipulation technique}

Electrical manipulation technique, such as single cell gel electrophoresis assay (SCGE) and dielectrophoresis (DEP) cell manipulation technique, also has been reported. SCGE was first presented by Ostling and Johanson in 1984, they used this method to study radiation-induced DNA damages in individual mammalian cells [30]. And the term "dielectrophoresis 


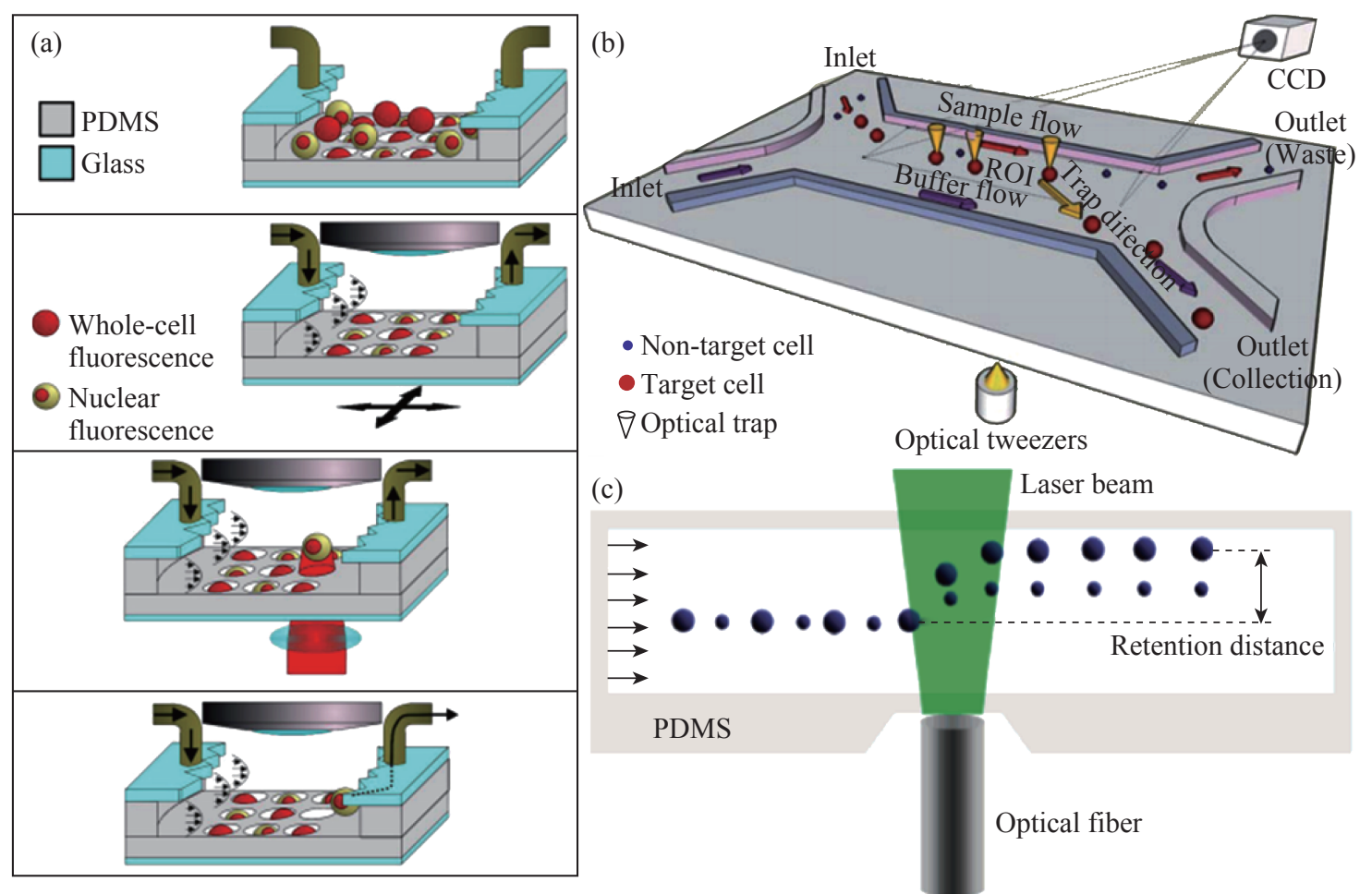

Fig. 3 Various structures of optical manipulation devices. (a) Each single cell is trapped in a microwell, selecting the target cell by employing fluorescence method, and then push the target cell out of the microwell by using a optical manipulation technique. (b) Cells solution and buffer are injected into the microfluidic by syringe pump, while the sample flow pass the detection zone, target cells are detected by CCD detector, the target cells will be changed direction by optical tweezers. (c) Cells are separated according to the size of cells by optical force [27-29].

(DEP)" was coined by Pohl in 1951, who performed important early experiments with small plastic particles suspended in insulating dielectric liquids and found that the particles would move in response to the application of a non-uniform AC or DC electric field [31]. At that time, however, the DEP manipulation technique had not been applied to manipulate bio-particles and cells. Currently, nevertheless, DEP manipulation technique has been highlighted due to its potential in the selective spatial manipulation of particles and cells.

SCGE was used as a tool to assess genetic damage in exposed populations. It enables the detection of various DNA damage in individual cells with ease and speed. It is, therefore, well suited to the analysis of a large group in a population [32]. Miloshev has applied the SCGE assay on yeast cells treated saccharomyces cerevisiae cells with hydrogen peroxide, methyl methanesulfonate (MMS) and two DNA damaging agents [2]. The human red blood cells were separated at the single-cell-level by using capillary zone electrophoresis in 2000 . This finding, which reported by Tsuda, is the first report to present the fine separation of population of red blood cells at single-cell-level [33]. However, SCGE is rarely found in single cell manipulation field, due to it is very difficult to manipulate individual cell at single-cell- level and hard to recovery single target cell.

At the same time, researchers developed DEP manipulation technique for single cell manipulation. DEP, the force produced by acting a non-uniform electric field upon a neutral object, is shown to be a simple and useful technique for study of cellular organisms [34]. The magnitude of DEP force is depended on the size, shape, electrical property of the particle, and the electric field gradient. While the direction of the DEP force is the same as the electric gradient, this phenomenon is called as positive-DEP (p-DEP). Conversely, it is called as negative-DEP (n-DEP). The DEP force can be expressed as Eq. (4),

$$
\left\langle\bar{F}_{D E P}(t)\right\rangle=2 \pi \varepsilon_{m} R^{3}\left[f_{C M}(\omega)\right] \nabla E_{R M S}^{2}
$$

where, $\varepsilon_{m}$ is the medium permittivity, $R$ is the particle radius and $E_{R M S}$ is the root mean square magnitude of the electric field. $f_{C M}$ is the Clausius-Mossotti (CM) factor. It is given by

$$
f_{C M}=\frac{\overline{\varepsilon_{p}-\bar{\varepsilon}_{m}}}{-\overline{\varepsilon_{p}}+2 \bar{\varepsilon}_{m}}
$$

where, $\bar{\varepsilon}_{p}$ and $\bar{\varepsilon}_{m}$ are the complex permittivity of 
the particle and the medium, respectively. And $\bar{\varepsilon}$ is expressed as $\varepsilon+\sigma / j \omega . \varepsilon$ is permittivity and $\sigma$ is conductivity of the particle and the medium, and $j=\sqrt{-1}$. The CM factor is comprised between -0.5 and 1 . When the particle is less polarizable than the surrounding medium $\left(f_{C M}<0\right)$ it will experience a n-DEP force pushing it towards regions of electric field minima. On the contrary, when the particle is more polarizable than the medium $\left(f_{C M}>0\right)$ it will be attracted by a p-DEP force towards regions of field maxima [35]. Based on the theory of DEP, scientists and engineers designed and developed many devices for manipulation cells. DEP cell manipulation technique is also a research highlight in cells manipulation field, due to the advantages of harmless to the cell, pre-treatment free, high precision, easy to manipulate an individual cell, and so on. Nowadays, DEP cell manipulation technique can achieve target cell separation [36], trapping/capturing [37] and release [38]. In order to obtain target particle or cell faster and more effective, a novel concept of active microwells, which can vertically trap and control single particles by means of n-DEP, was presented. The authors applied sinusoidal signals on the electrodes at frequencies ranging from $100 \mathrm{kHz}$ to $1.5 \mathrm{MHz}$ and amplitudes between $2 \mathrm{~V}$ and $7 \mathrm{~V}$. Particles are successfully trapped and levitated at the level of the central electrode in the middle of microwells with a diameter of $125 \mu \mathrm{m}$, shown in Fig. 4 [35].

Different electrode shapes produce different electric field gradient. Therefore, various electrode shapes were designed for cells and particles manipulation, such as ring electrode, quadrupole and so on. Thomas designed a ring electrode for single cell immobilization, shown in Fig. 5(a) and (b). The analysis of the 80 $\mu \mathrm{m}$ diameter ring electrode showed that a $15.6 \mu \mathrm{m}$ diameter latex particle could be held with a force of $23 \mathrm{pN}$ when applied voltage of $5 \mathrm{~V}$ peak-peak on the electrode [39]. Another design using quadrupole to trap cells by means of alternating current electrothermal effect (ACET), shown in Fig. 5(c) and (d) [40]. This quadrupole method can trap single cells by adding voltage on different electrode groups.

Park presented a design, fabrication, and characterization of a microfluidic biochip with integrated actuation electrodes for manipulation single cell and single micro-bead by DEP and sensing electrodes for detection the trapping particles by using the impedance detection method, shown in Fig. 6 [41].

DEP cell manipulation technique combines with microfluidic, which contains a microwell array inside, for high-efficient single cell trapping and analysis was reported in 2011 [42]. At the same time, a novel concept of micro-cavity array with multilayer electrodes for trapping and programmable releasing single cells was proposed [38, 43-45]. The model of multilayer electrodes DEP chip is shown in Fig. 7(a). This design can trap cells by applying the AC signal to the middle and top electrodes, the electric field gradient was simulated by finite element analysis software, as shown in the Fig. 7(b). Similarly, applied the AC signal to the bottom and top electrodes for releasing cells, and the electric field gradient is shown in Fig. 7(c).

To fabricate large electrode array in microfluidic chip, maybe result in a complex and large electrode driver circuit. This will need to integrate both MEMSand CMOS-process, and lead to very high design costs.

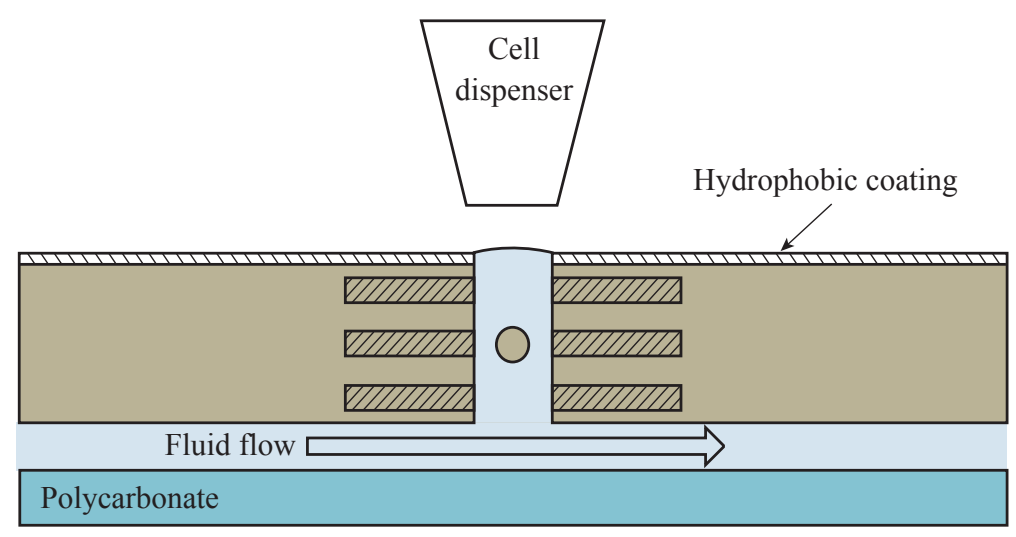

Fig. 4 Cross-section of a microwell consisting of a $125 \mu \mathrm{m}$ hole drilled in a multilayer flexible PCB substrate. A fluid carrier is used to create a channel under the well which is filled by capillarity, while the hydrophobic coating on top side allows the fluid to be kept within the hole and a meniscus consequently to form. A particle can be inserted manually or with the support of a cell dispenser from the top and will be trapped at the level of the second metal layer [35]. 

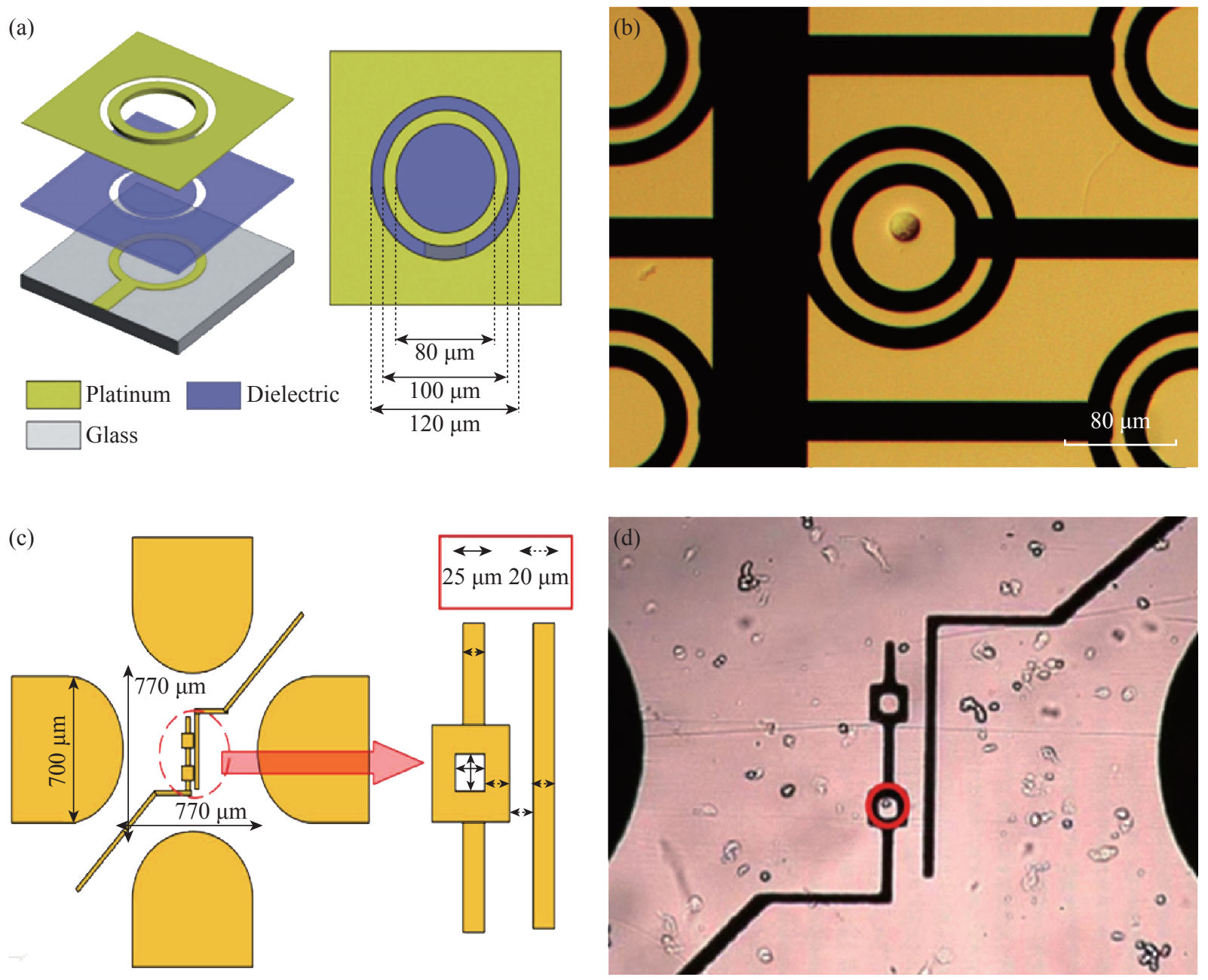

Fig. 5 Different electrode shapes for cell manipulation. By applying voltage on the different electrodes to generate non-uniform electric field for cells trapping $[39,40]$.
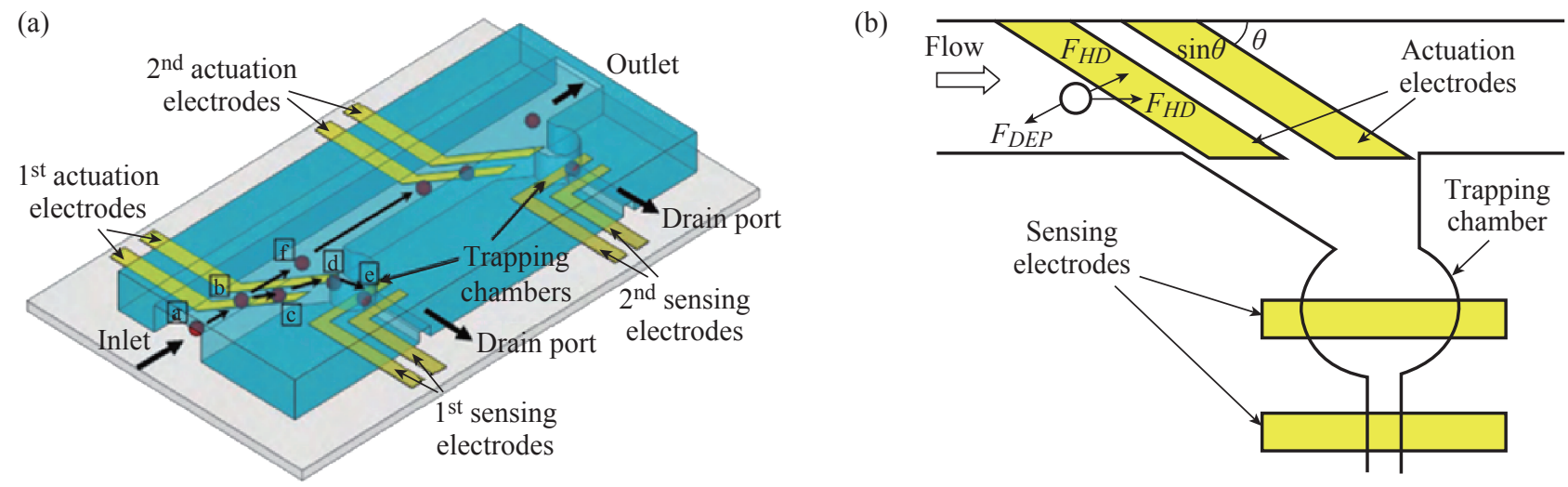

Fig. 6 (a) Schematic diagram of proposed microfluidic chip; (b) Forces act on a particle, causing the particle moving into the trapping chamber for analyzing [41].

Hence, researchers proposed optoelectronic tweezers (OETs) based on the DEP principle. OETs devices are conceptually similar to solar cells. Instead of generating photocurrents, the photo generated carriers increase the conductance locally near the illuminated area, thus forming virtual electrodes, the experimental and fundamental structure of OETs shown in Fig. 8
[14].

On the OETs basis, a massively parallel manipulation of single cells and micro-particles method was presented. It has been demonstrated that parallel manipulation of 15,000 particles can be trapped on a $1.33 \times 1.0 \mathrm{~mm}^{2}$ area [46]. A few years later, the authors designed a device of phototransistor-based OETs for 


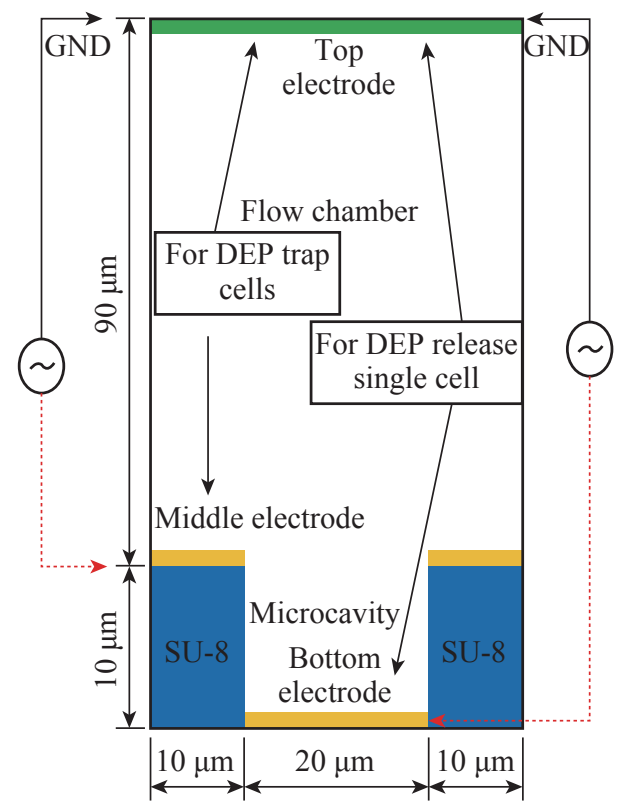

(a)

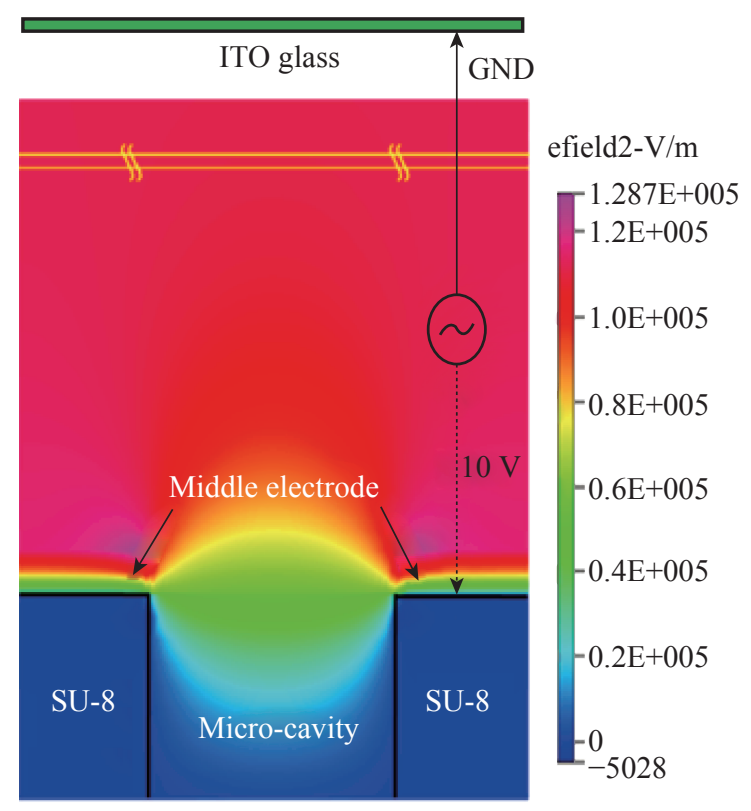

(b)

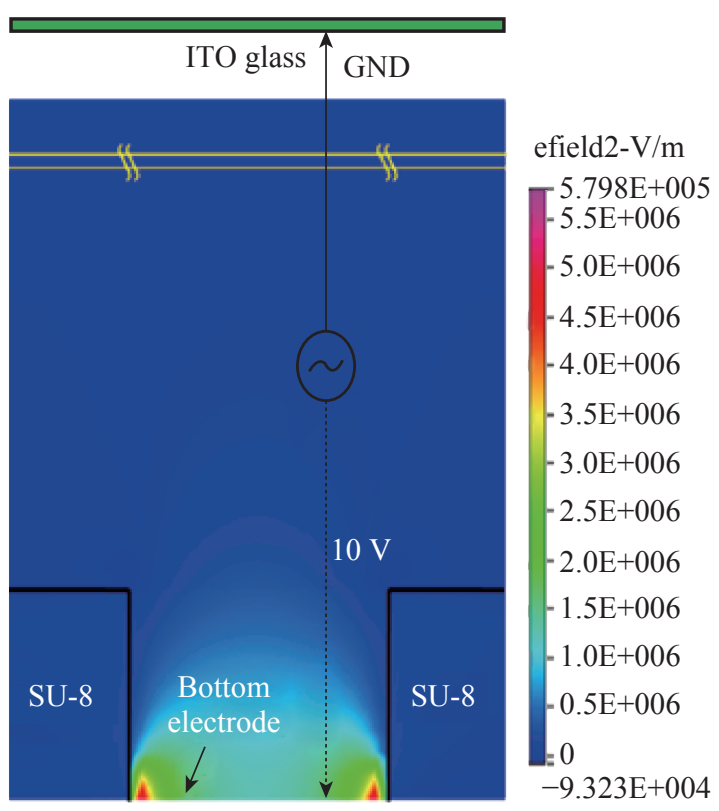

(c)

Fig. 7 Model and simulation result of multilayer electrodes EDP chip. (a) In this design, by applying a AC voltage on top and middle electrodes for trapping cells into microwell, and by applying a AC voltage on top and bottom electrodes to release cell out of microwell. (b)-(c) shown the gradient of electric field generate by applying voltage on different electrodes group [38, 43-45].

dynamic cell manipulation in cell culture media. This device demonstrated precise control of separation between two cells [47]. In order to obtain a single cell for sample preparation and analysis, Huang designed an OETs platform with microfluidic [48]. Recently, a compact OETs system combined cell manipulation and analysis is presented. The trap strength and profile for two emission wavelengths have been measured and the maximum trapping force of 13.1 and $7.6 \mathrm{pN}$ was achieved by projected micro-LED devices emitting at $\lambda_{\max } 520 \mathrm{~nm}$ and $450 \mathrm{~nm}$, respectively [49]. In all, this OETs system is very suited for single cell manipulation and fluorescence imaging of immune cell.

\section{Magnetic manipulation technique}

Magnetic manipulation technique, which known as magnetic tweezers, was emerged later than electrical and optical manipulation techniques. The magnetic force is generated by a magnetic field gradient. While this force acting on a magnetic particle, it depends 


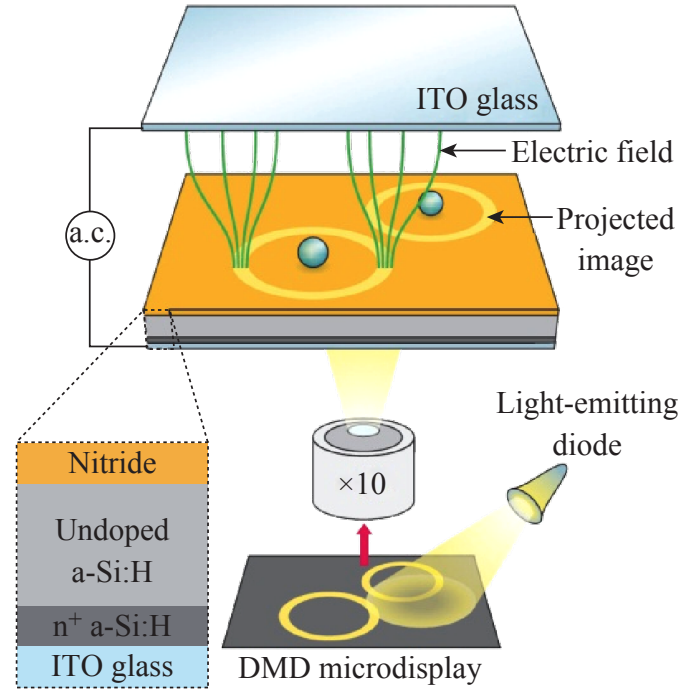

Fig. 8 A experimental and fundamental structure of Optoelectronic tweezers. Liquid-containing particles are sandwiched between a transparent electrode and a photoconductive electrode. Light patterns from a digital projector are image onto the device. The image together with an a.c. electrical bias generates 'virtual electrodes' that in turn create dielectrophoretic trap in the illuminated areas [14].

on the volume of the particle $(V)$, the difference in magnetic susceptibilities, $\Delta \chi\left(\Delta \chi=\chi_{p}-\chi_{m}\right)$, between the particle $\left(\chi_{p}\right)$ and surrounding buffer medium $\left(\chi_{m}\right)$, as well as the magnetic flux density $(B)$ and magnetic flux density gradient $(\nabla B)[50,76]$,

$$
F_{\mathrm{m}}=\frac{V \cdot \Delta \chi}{\mu_{0}} B \cdot \nabla B
$$

To our knowledge, there are two ways to create magnetic field. One way is applying permanent magnets to create magnetic field, and the other way is utilizing electromagnets. A system that utilizing electromagnets is more flexible and easier controlled than the permanent one.

Based on this theory, the magnetic manipulation technique for particles was proposed. This technique with great advantages, for instance, magnetic manipulation technique is not affected by particle surface charges, $\mathrm{PH}$, ionic concentrations or temperature. In recent years, it was widely applied in biology, such as to manipulate DNA or RNA molecules [51], micro- or nanoparticles [52], single cell [53], and can be used for analysis of cell mechanics as well $[54,55]$. In order to instead of permanent magnets and ease to control the particles, an electromagnet was assembled to generate a constant magnetic gradient for DNA manipulation by Haber [56]. After that, based on the general circuit theory and magnetic bubble technology, Lim demonstrated that a class of integrated circuits for executing sequential and parallel timed operations on an ensemble of single particles and cells [57]. In single cell manipulation field, Liu reported a simple and straightforward approach to fabricate magnetic nanofiber segments for cell manipulation. They used NIH 3T3 cells, which were cultured in a medium containing magnetic fibers, as assay samples, the result of the assays showed that cells can be conveniently manipulated with a magnet, shown in Fig. 9 [58].

Ebrahimian injected magnetic particles of $1 \mu \mathrm{m}$ diameter into barley cell vacuoles using a microinject system under microscopic control. To meet the purpose of manipulation cells at single-cell-level by using magnetic tweezers to manipulate the magnetic particles in the cells [59]. In order to fabricate a microstructure that can be powered and controlled wirelessly in fluidic environments. A kind of biocompatible ferromagnetic microtransporters driven by external magnetic fields was described by Sakar. Those microtransporters were fabricated with SU-8 photoresist and magnetic nanoparticles [60]. Fig. 9(a) shows the shapes of the microtransporters. Among Fig. 10, (b) to (d) show the process of manipulation of a target cell. (b) The orientation of the transporter is adjusted according to the position of the target cell. (c) With the application of an out-of-plane time-varying magnetic field, the transporter starts translating toward the target. The pulsing frequency is $100 \mathrm{~Hz}$. (d) The target is engaged and transported out of the field of view. At the end, the authors demonstrated that the average velocity of the transporter can up to $350 \mu \mathrm{m} / \mathrm{s}$.

Another application of magnetic manipulation technique is magnetic activated cell sorting (MACS). MACS is a method for separation of various cell populations depending on their particular surface antigens. The procedure of MACS is that the cell solution, which the target cells expressing their antigen to attach to the magnetic nanoparticles coated antibodies, is transferred on a column placed in a strong magnetic field, the labeled cells are separated by the magnetic force from the magnetic nanoparticles. MACS method is extensively used in biotechnology for a wide range of application from in vitro diagnostics to cell-based therapies. MACS allow high-throughput separation of magnetically labeled target species [61]. Nowadays, circulating tumor cells (CTCs) have been studied as a mean of overcoming cancer. In order to select and isolate the rarity and heterogeneity of CTCs, a two-stage microfluidic chip was designed by Hyun, 
(a)

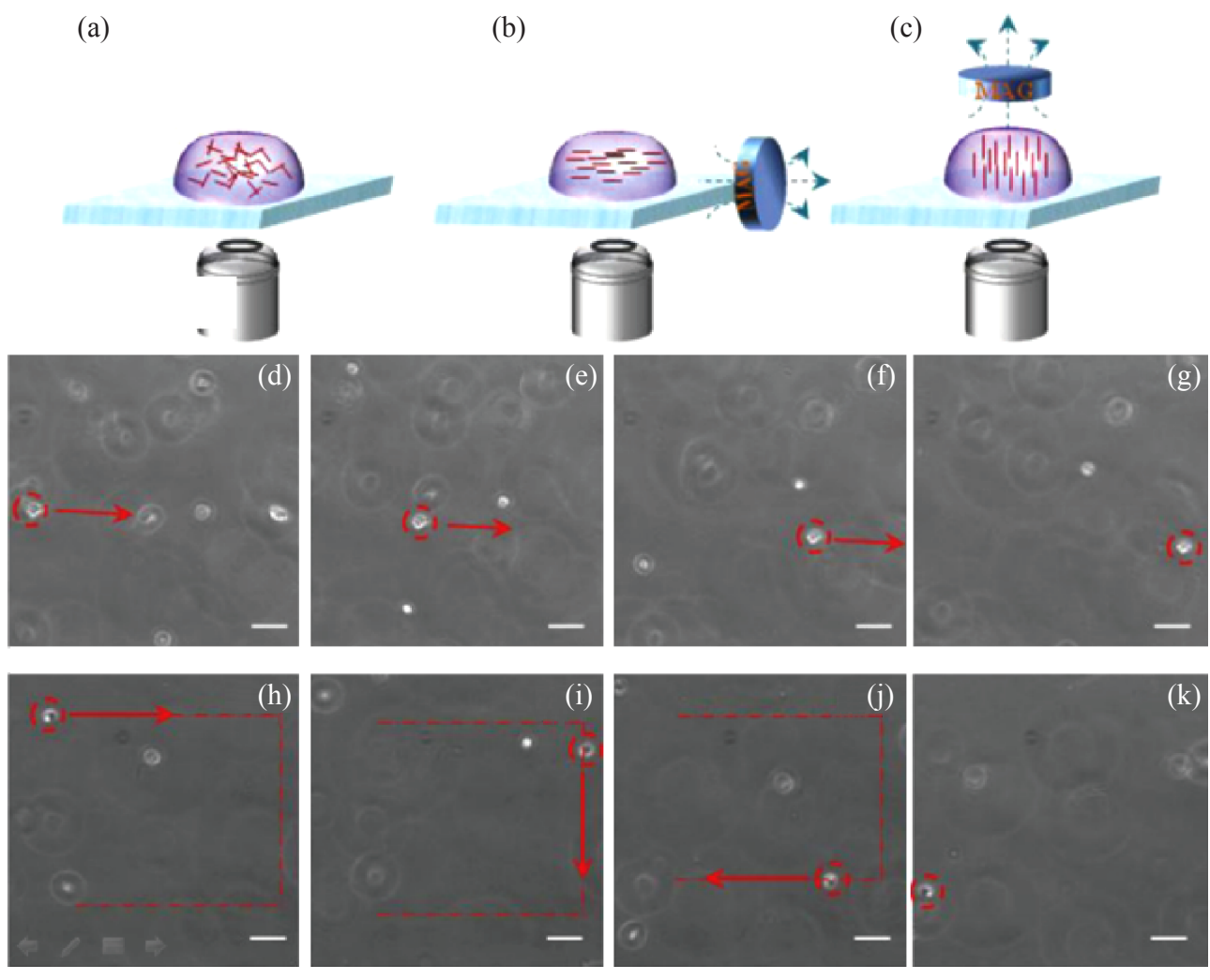

Fig. 9 (a)-(c) Schematic of magnetic particle doped nanofiber segments in water drops on a glass slide. (d)-(k) Showing cell movement path, controlled by an external magnetic field. The scale bar is $20 \mu \mathrm{m}$ [58].

(a)
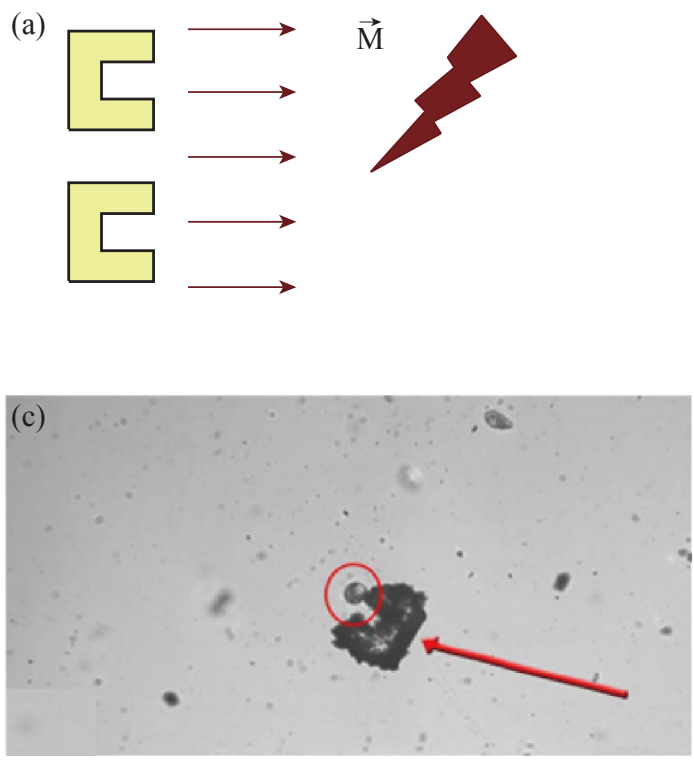
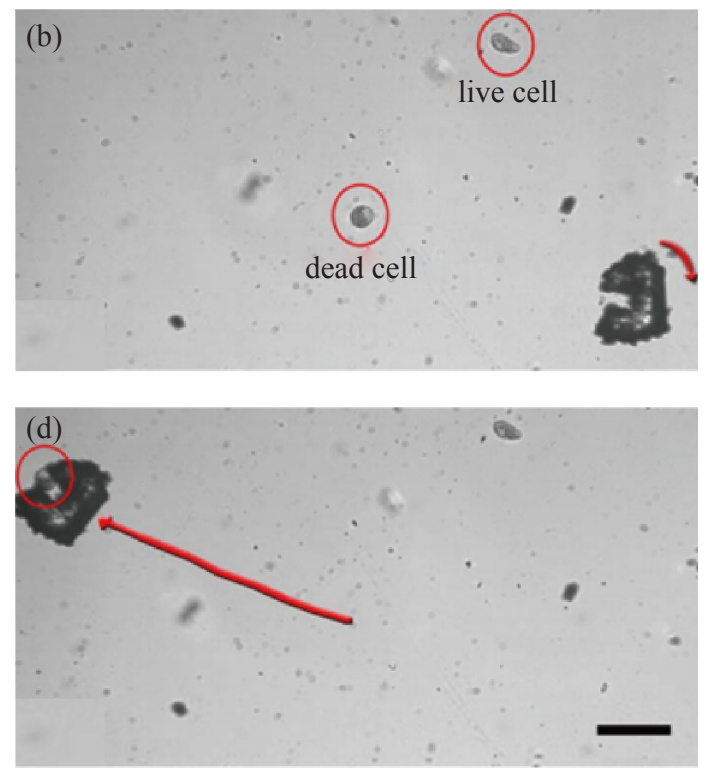

Fig. 10 (a) The shapes of microtransporter. (b)-(d) Process of manipulation a target cells. (b) By controlling direction of the magnetic field to move the micotransporter towards the dead cell. (c) Moving the microtransporter to capture the dead cell by magnetic flied. (d) Moving away the dead cell by microtransporter. The scale bar is $100 \mu \mathrm{m}[60]$.

[62]. The first stage (Fig. 11(a) and (b)) involves a microfluidic MACS chip to elute white blood cells (WBCs). The second stage (Fig. 11(c) and (d)) involves a geometrically activated surface interaction (GASI) chip for the selective isolation of CTCs.

Due to the physics of separation is based on a single parameter - magnetization, the MACS is generally effective only for single-species-target cells selection. In order to separate multi-species cells, researchers according to difference magnetization degree of different types of magnetic nanoparticle and base on the principle of MACS, a multi-target magnetic 

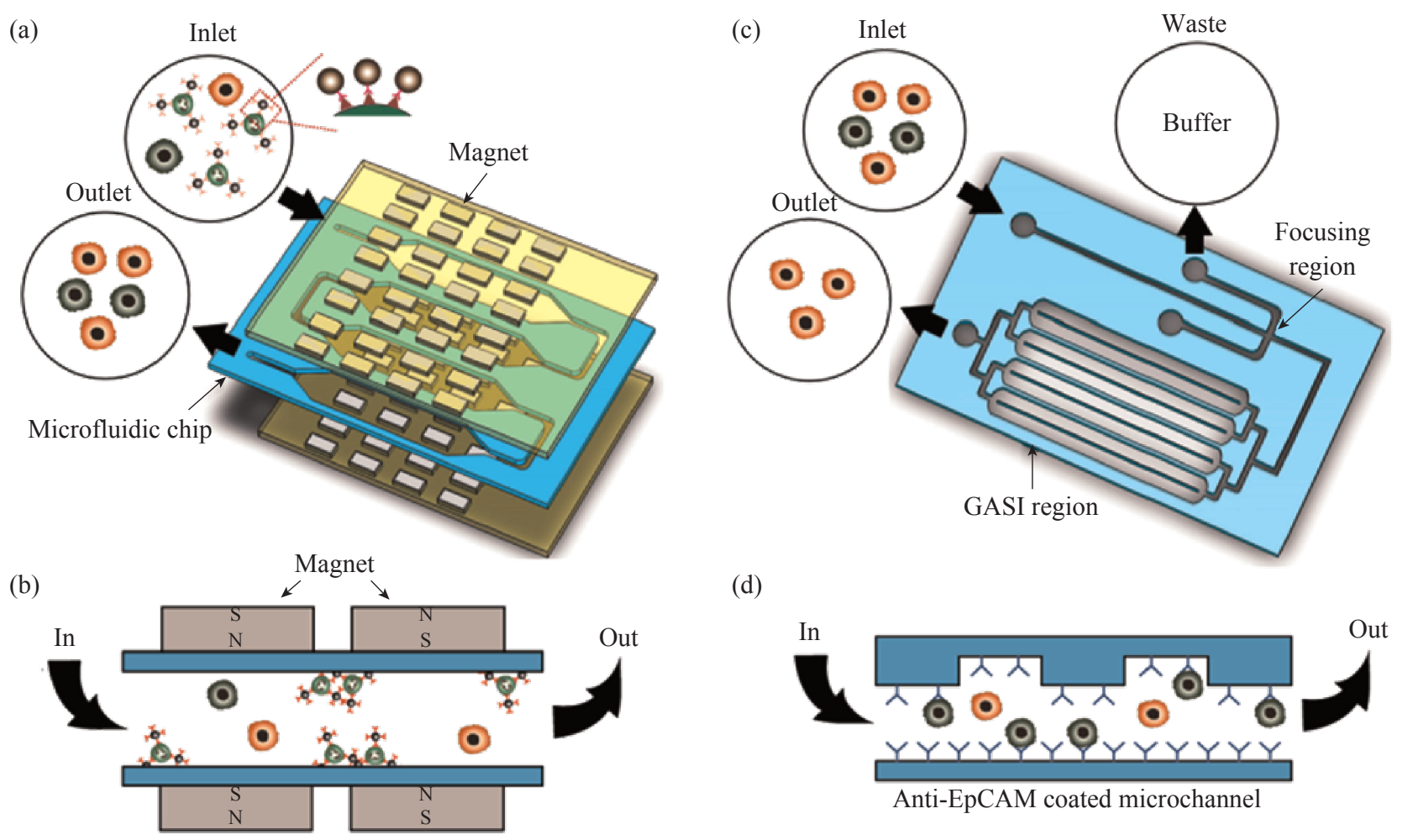

(d)

\section{OCTC (EpCAM+) $\bigcirc \mathrm{CTC}\left(\mathrm{EpCAM}^{-}\right)$- White blood cell $\curlyvee_{\text {Magnetic nano particle }}^{\mathrm{CD} 45 \text { antibody conjugated }}$}

Fig. 11 (a) Schematic diagram of a two-stage enrichment chip for isolation of CTCs. (b) White blood cells are trapped by magnetic field. (c) Microfluidic structure for CTCs separation. (d) CTCs(EpCAM+) are trapped by the antibody [62].

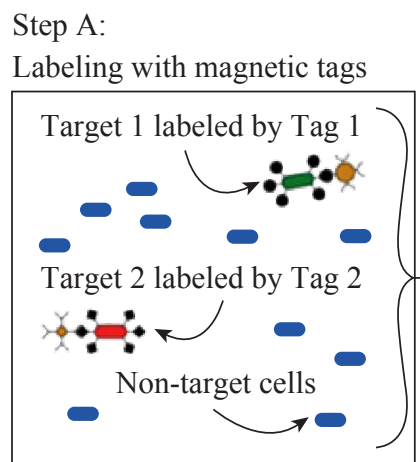

Step B:

Multi-target magnetophoresis

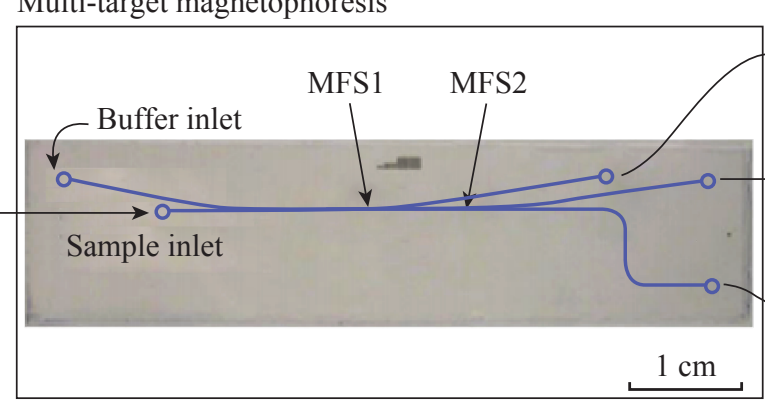

(a)

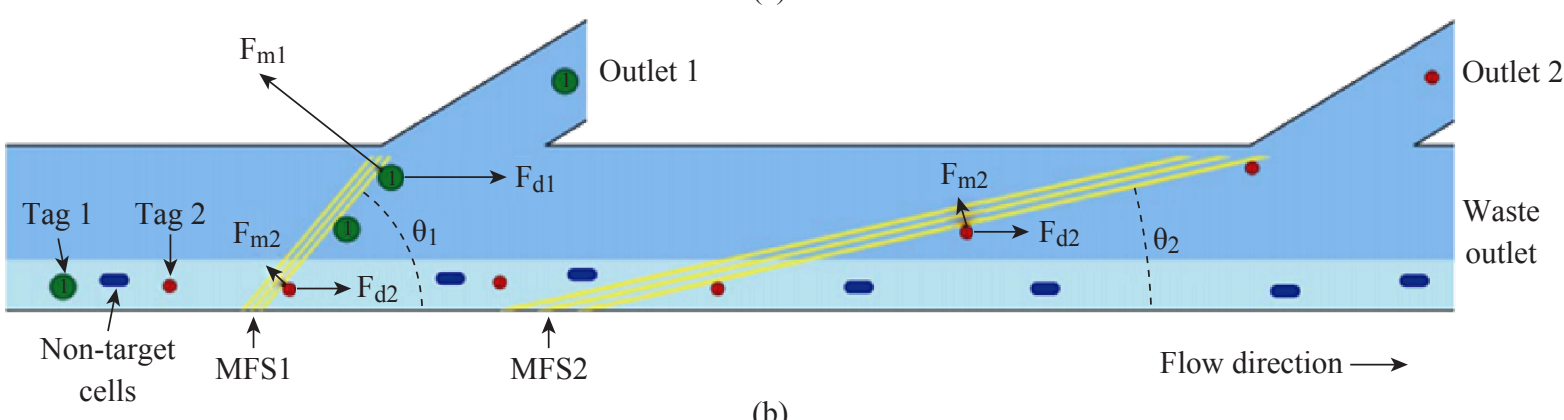

(b)
Step C:

Quantification via cytometry
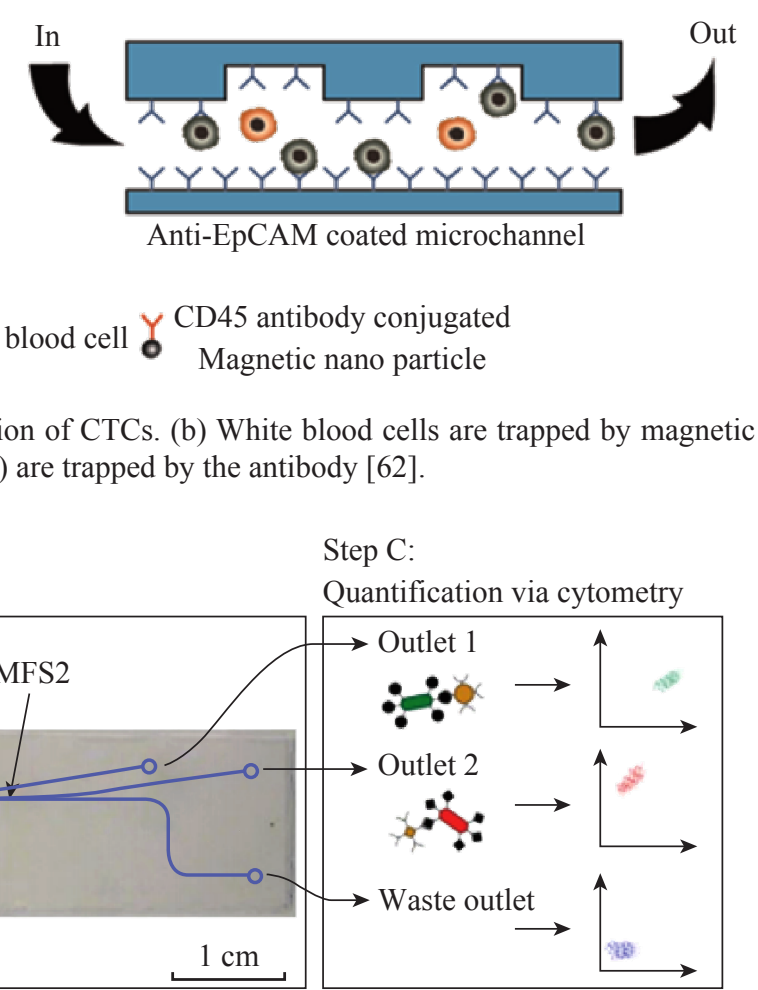


\section{Acoustic manipulation technique}

In recent years, surface acoustic wave (SAW) techniques in microfluidic were concerned by researchers. Recent research demonstrates that SAW technique can be an effective method to manipulate micro-scale particles [64]. The best-known of SAW is composed of a longitudinal and a vertically polarized shear component [65]. SAWs are generally produced by applying an appropriate voltage on the electrodes of piezoelectric material. The electrodes will generate propagating mechanical stress. A typical SAW device uses at least one set of metallic interdigital transducers (IDTs) fabricated on the surface of a piezoelectric substrate. The IDT then introduces the electric field, generating a SAW displacement amplitude on the order of $1 \mathrm{~nm}$ [66].

In last few years, SAW technology combined with microfluidic technique has turned into a tool, which known as acoustic tweezers, to manipulate particles. Liquid inside of the microfluidic is pressed by mechanical stress. Hence, a liquid pressure gradient in microfluidic can be controlled by controlling the IDTs. Based on this principle, a continuous particle separation in a microfluidic channel via standing surface acoustic waves was introduced by Shi, shown in Fig. 13 [67]. The particle separation method is capable of separating virtually all kind of particles with high separation efficiency and low power consumption, the separation mechanism and result of separation.

After a short while, a design of on-chip manipulation of single particles, cells, and organisms using SAW was presented by Ding [68]. Fig. 14 shows the (a) schematic illustrating of a microfluidic device with orthogonal pairs of chirped IDTs for generating standing SAW. (b) shows standing SAW field generated by driving chirped IDTs at frequency $f_{1}$ and $f_{2}$. When particles are trapped at the nth (it is an ordinal number) pressure node, they can be translated a distance of $(\Delta \lambda / 2)$ n by switching from $f_{1}$ to $f_{2}$. This relationship indicates that the particle displacement can be tuned by varying the pressure node where the particle is trapped. (c) shows simulated pressure field between adjacent pressure anti-nodes. (d) shows the author used a 10- $\mu \mathrm{m}$ fluorescent polystyrene bead to write the word "PNAS" and dynamic control of a bovine red blood cell to trace the letters "PSU".

\section{Mechanical manipulation technique}

Using mechanical methods to manipulate particles
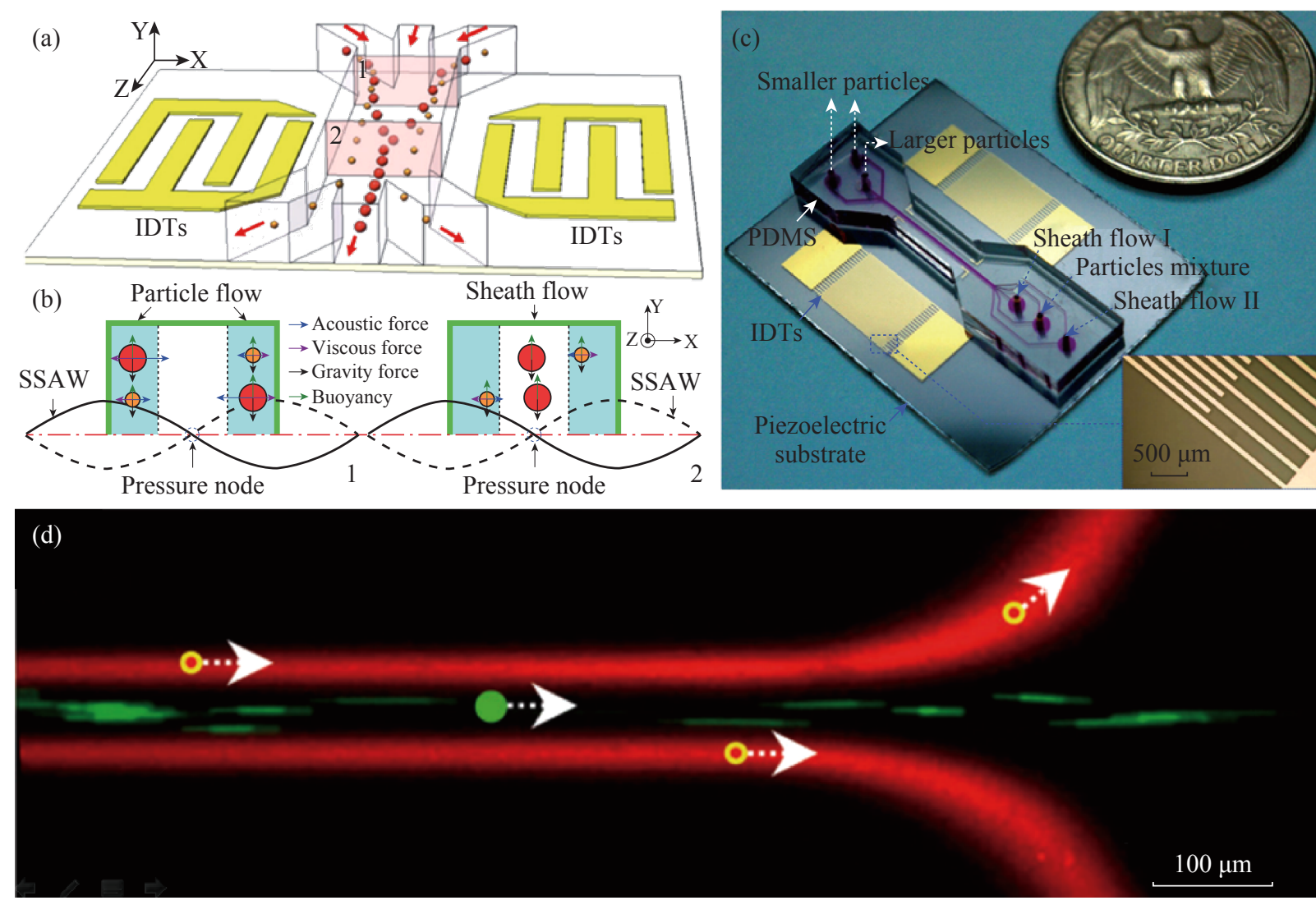

Fig. 13 (a)-(b) Schematic of separation mechanism, (c) Optical image of the design, (d) Image of particles separation [67]. 

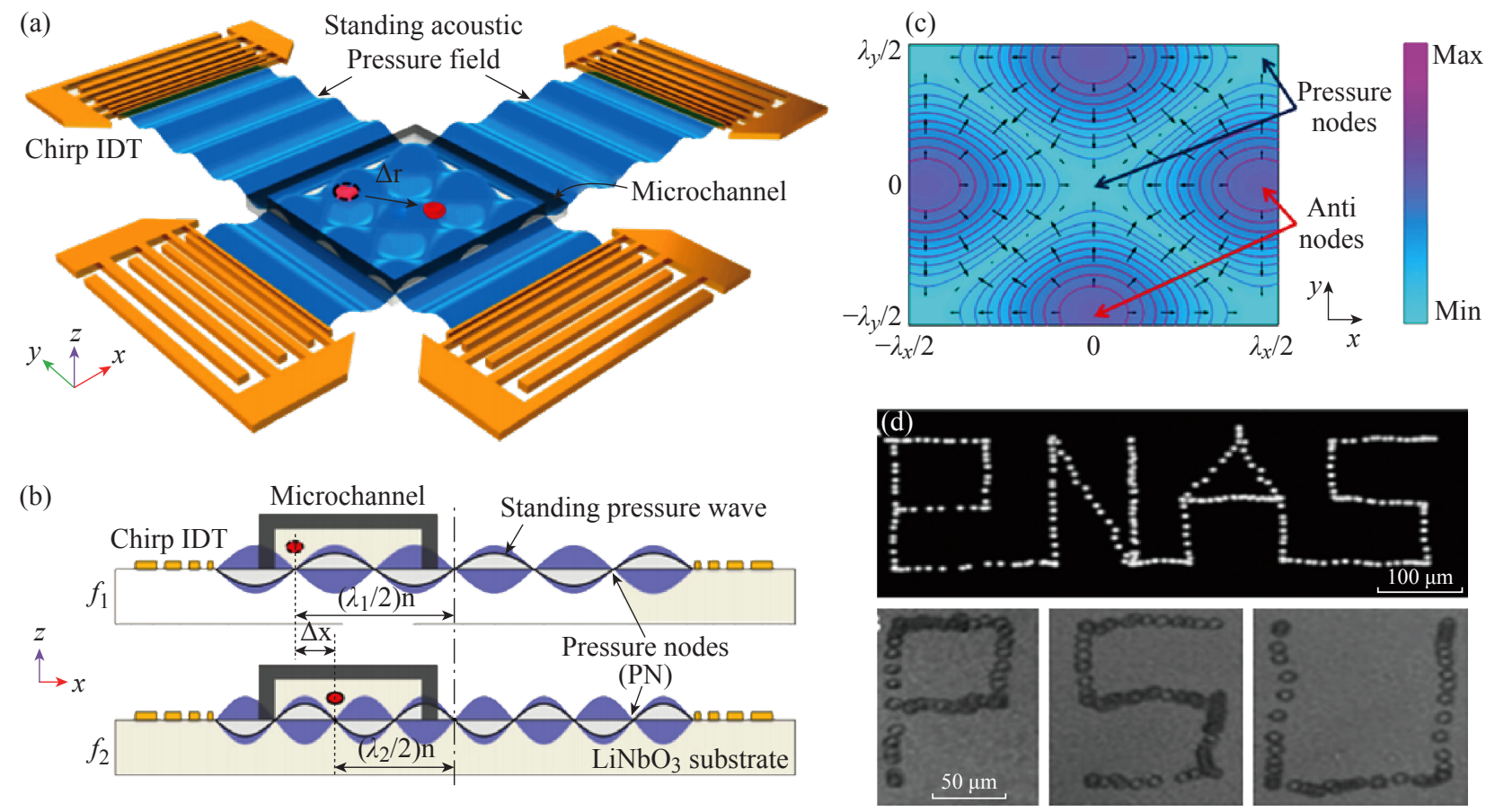

Fig. 14 (a)-(b) Device structure and working mechanism of the acoustic tweezers. (c) The pressure nodes of solution inside this device, the particles will move to and stay at the minimum pressure nodes. (d) Result of polystyrene bead and cell manipulation, top one using polystyrene beads to write 'PNAS' letters and the bottom one employing cells to write 'PSU' letters [68].
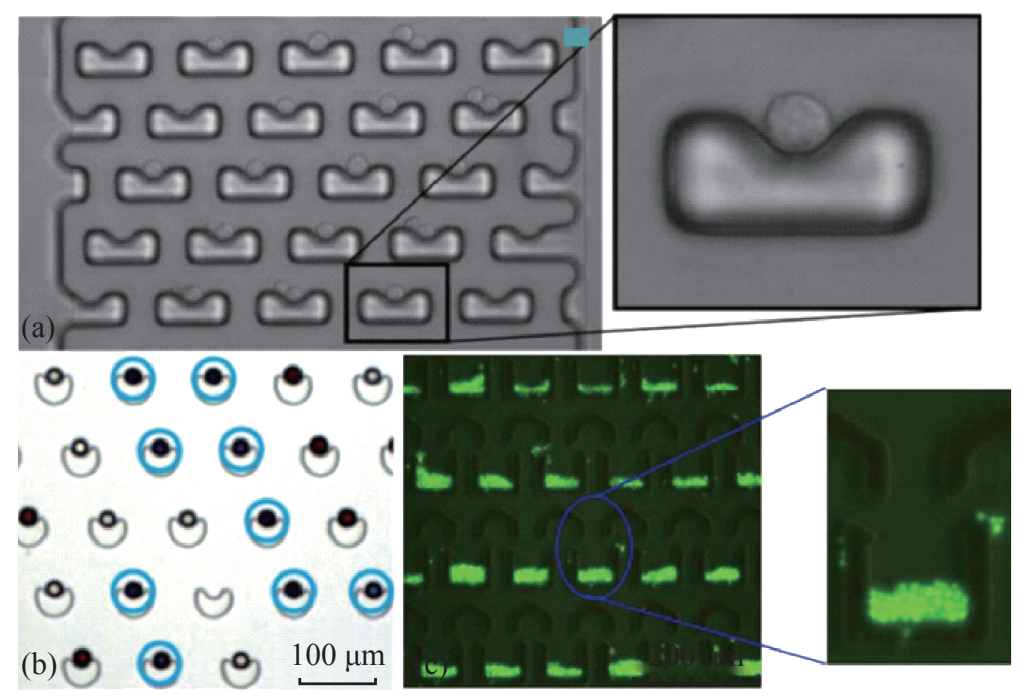

(d)

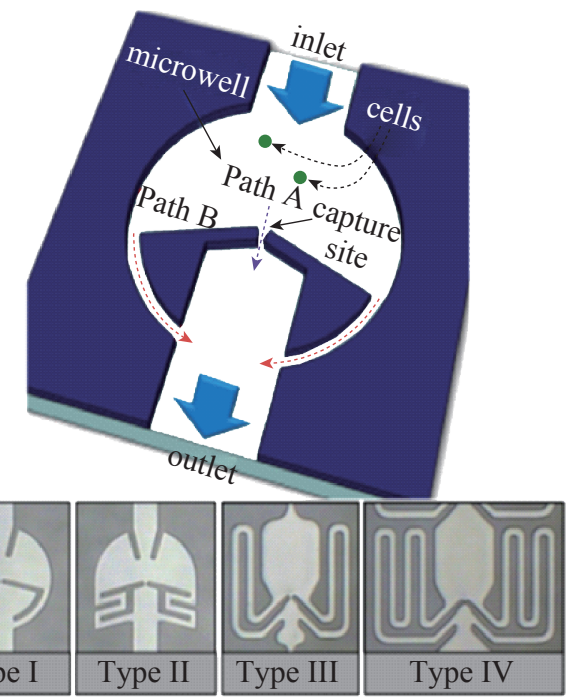

Fig. 15 Different shapes for cells capture. (a) Cells trapped in U-shape structures. (b) Cells trapped in V-shape. (c) Cells trapped in U-shape with Y-shaped fluidic guide. (d) Four types of different structure for cells trapping. [70-73].

and bio-particles, such as capturing, sorting, and isolation have been emerged several decades. These methods use mechanical forces, such as gravity, hydrodynamic, and suction to manipulate particles or bio-particles. Different microwell dimension arrays for large-scale single cell trapping were designed by Rettig [69]. With this method, the parameters that maximize single-cell occupancy for two cell types, including microwell diameter, microwell depth, and settle time, were determined. The authors injected cell suspension into the microfluidic, which contains microwell array inside, and then the cells will settle into the microwells due to gravity. They found that microwell with an aspect ratio of $\sim 1$ yield optimal single-cell occupancy. Other structures for cell manipulation base on hydrodynamic also were presented. A device consists of physical U-shape hydrodynamic trapping structures array for single cell trapping and culture had been designed, the shape of this design is shown in Fig. 15(a) [70]. Researchers improved the trapping structure for high-efficient single cell capture. In order to trap particles more efficient, an geometrical V-cup 
barriers array was presented. The capture efficiency of this platform is nearly $100 \%$, and the trapping shape is shown in Fig. 15(b) [71]. Another structure for cells capture was proposed by Chen [72]. In this design, the first layer consists of spacers to create small gap between the upper layer and glass. The second layer is a sharp corner U-shaped compartment with sharp corners at the fore-end. And a Y-shaped fluidic guide structures are designed on the top of each U-shaped capture structures, shown in Fig. 15(c). A highly efficient single cell capture scheme using hydrodynamic guiding structures also was presented [73]. The authors designed four types of cell capture module and tested for optimal structure. The capturing efficient of this single-cell capture chip is more than $80 \%$ shown by the experiments. The structures for single cell trapping were shown in Fig. 15(d).

The structures were continually improved by researchers, however, those designs only suit for one or several kind of cells due to its manipulation principle depend on both geometric size of particles and capture structures. Thus, the method of suction for manipulation cell was presented. A micromanipulation method for single prokaryotic cells extracting was improved, shown in Fig. 16 [74]. Fig. 16(a) shows
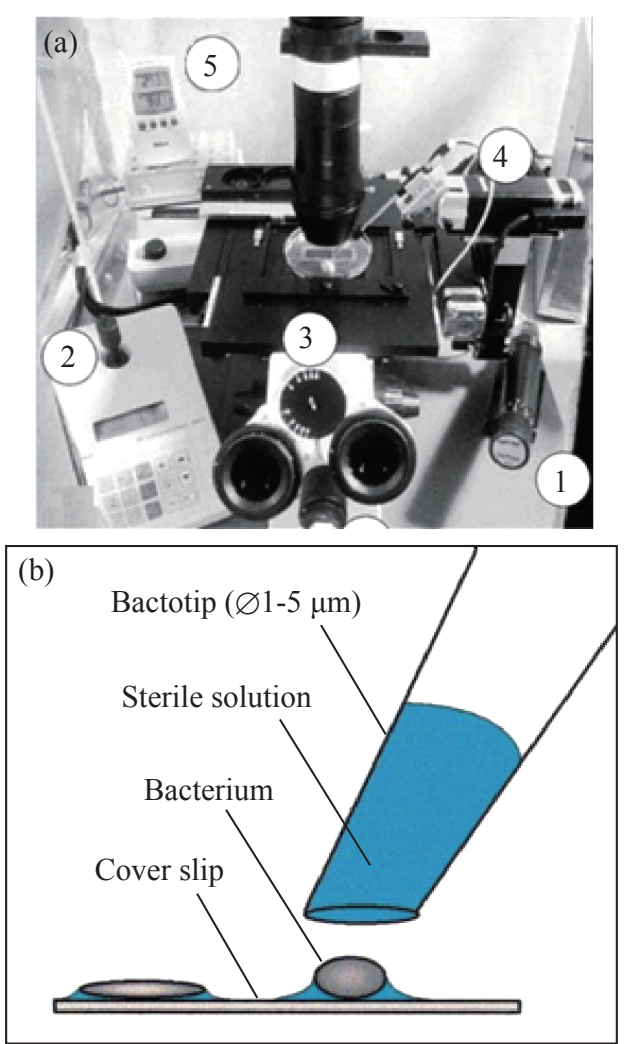

the workstation for manipulation of single cells, (1) CellTram Oil, (2) joystick, (3) inverse scope, (4) micromanipulator, (5) thermometer/hygrometer. (b)(d) shows the schematic drawing of the isolation of a single bacterial cell.

Another design based on the method of suction was presented by Anis and his colleagues [75]. They developed a pico-liter pump and integrated into a robotic manipulation system. This pico-liter pump can automatically select and transfer individual living cells of interest to analysis locations. The authors demonstrated that the pump aspirates and dispenses volumes of fluid between $500 \mathrm{pL}$ and $250 \mathrm{~nL}$ at flow rates up to $250 \mathrm{~nL} / \mathrm{s}$. And then they successfully accomplished single cell manipulation assay by using Barrett's esophagus cell. The six-axis robotic workstation schematic and assay image shows in Fig. 17.

Furthermore, Flanders team presented a methodology for fabrication of cellular force sensors composed of high aspect ratio cantilevered poly (3, 4-ethylene dioxythiophene) (PEDOT) fibers [77]. These fiber sensors can be used to characterize the dynamics of apical pseudopod-substrate adhesive contacts of $D$. discoideum cells. They also shown that
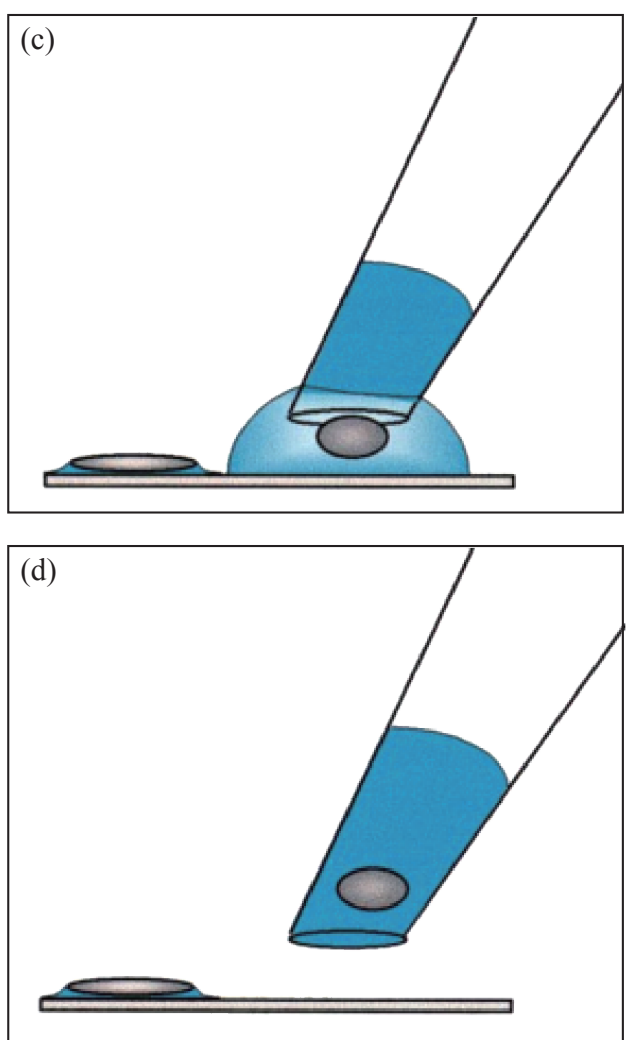

Fig. 16 (a) A workstation and schematic drawing for isolation a single bacterial cell. (b)-(d) The procedure of isolation a target cell. $[74]$. 
(a)

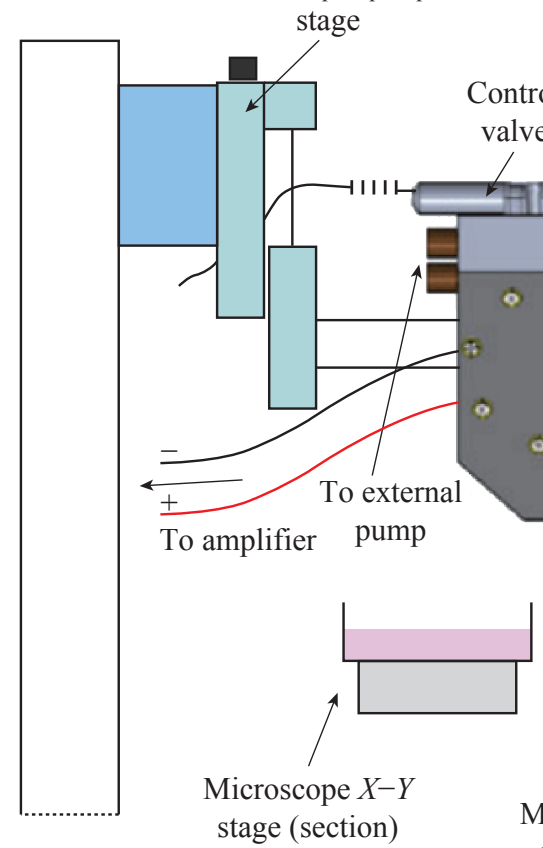

Illuminator and condenser

Pump assembly

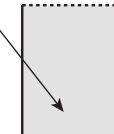

\section{$\square$}


Table 1 Comparison of each cell manipulation technique

\begin{tabular}{|c|c|c|c|}
\hline \multicolumn{2}{|c|}{ Manipulation technique } & Advantages & Drawbacks \\
\hline \multicolumn{2}{|c|}{ Optical } & $\begin{array}{l}\text { High precision } \\
\text { High stability } \\
\text { High throughput }\end{array}$ & $\begin{array}{l}\text { May harm cells } \\
\text { Device complexity } \\
\text { Device expensive }\end{array}$ \\
\hline \multirow[b]{2}{*}{ Electrical } & SCGE & $\begin{array}{l}\text { Harmless to cells } \\
\text { Device simple }\end{array}$ & Hard to manipulate cells in single-cell-level \\
\hline & DEP & $\begin{array}{l}\text { Harmless to cells } \\
\text { High throughput } \\
\text { High precision } \\
\text { Device simple }\end{array}$ & $\begin{array}{c}\text { Hard to control } \\
\text { Drive circuit is complex }\end{array}$ \\
\hline \multicolumn{2}{|c|}{ Magnetic } & $\begin{array}{l}\text { High precision } \\
\text { Device simple }\end{array}$ & $\begin{array}{c}\text { Magnetic material pre-label } \\
\text { Hard to control }\end{array}$ \\
\hline \multicolumn{2}{|c|}{ Acoustic } & $\begin{array}{c}\text { Harmless to cells } \\
\text { Low power consumption } \\
\text { Device is simple }\end{array}$ & $\begin{array}{l}\text { Hard to control } \\
\text { Electrode drive is complex }\end{array}$ \\
\hline \multirow{3}{*}{ Mechanical } & gravity & Device simple & Only use for trapping \\
\hline & hydrodynamic & Device simple & Hard to manipulate cells in single-cell-level \\
\hline & suction & Simple to operate & $\begin{array}{l}\text { Device is complex } \\
\text { Hard to align }\end{array}$ \\
\hline
\end{tabular}

(Project no: 15441904800)

\section{Reference}

[1] H. Yun, K. Kim, W.G. Lee, Cell manipulation in microfluidics. Biofabrication. 2013, 5(2): 022001.

[2] G. Miloshev, I. Mihaylov, B. Anachkova, Application of the single cell gel electrophoresis on yeast cells. Mutation Research. 2002, 513(1-2): 69-74.

[3] J.L. Zabzdyr, S.J. Lillard, New approaches to single-cell analysis by capillary electrophoresis. Trends in Analytical Chemistry. 2001, 20(9): 467-476.

[4] J. Chen, D. Chen, T. Yuan, et al., A microfluidic chip for direct and rapid trapping of white blood cells from whole blood [J]. Biomicrofluidics. 2013, 7(3): 34106.

[5] S.W. Lee, J.Y. Kang, I.H. Lee, et al., Single-cell assay on CD-like lab chip using centrifugal massive single-cell trap. Sensors and Actuators A: Physical. 2008, 143(1): 64-69.

[6] Y. Wakamoto, I. Inoue, H. Moriguchi, et al., Analysis of single-cell differences by use of an on-chip microculture system and optical trapping. Fresenius' Journal of Analytical Chemistry. 2014, 371(2): 276-281.

[7] G.M. Whitesides, The origins and the future of microfluidics. Nature. 2006, 442(7101): 368-373.

[8] I. Pushkarsky, P. Tseng, C. Murray, et al., Research highlights: microfluidics and magnets. Lab on a Chip. 2014, 14(16): 2882.

[9] D. Di Martino, G. Giuffre, N. Staiti, et al., Single sperm cell isolation by laser microdissection. Forensic science international. 2004, 146 Suppl: S151-S153.

[10] A.B. Fuchs, A. Romani, D. Freida, et al., Electronic sorting and recovery of single live cells from microlitre sized samples. Lab Chip. 2006, 6(1): 121-126.

[11] W.E. Huang, A.D. Ward, A.S. Whiteley, Raman tweezers sorting of single microbial cells. Environmental microbiology reports. 2009, 1(1): 44-49.

[12] R.B. Keithley, M.P. Metzinger, A.M. Rosado, et al., Manipulating ionic strength to improve single cell electrophoretic separations. Talanta. 2013, 111: 206-214.

[13] X. Wong, C. Rosales, Robust dielectrophoretic singlecell trap design using BEM. Engineering Analysis with
Boundary Elements. 2008, 32(5): 388-394.

[14] M.C. Wu, Optoelectronic tweezers [J]. Nature Photonics. 2011, 5(6): 322-324.

[15] A. Ashkin, Acceleration and Trapping of Particles by Radiation Pressure. Physical Review Letters. 1970, 24(4): 156-159.

[16] A. Ashkin, J.M. Dziedzic, Optical trapping and manipulation of viruses and bacteria. Science. 1987, 235(4795): 1517-1520.

[17] K.C. Neuman, S.M. Block, Optical trapping. Review of Scientific Instruments. 2004, 75(9): 2787-2809.

[18] D.G. Grier, A revolution in optical manipulation. Nature. 2003, 424(14): 810-816.

[19] M. Goksör, J. Enger, D. Hanstorp, Optical Manipulation in Combination with Multiphoton Microscopy for SingleCell Studies. Applied Optics. 2004, 43(25): 4831-4838.

[20] K.O. Greulich, G. Pilarczyk, A. Hoffmann, et al., Micromanipulation by laser microbeam and optical tweezers: from plant cells to single molecules. Journal of Microscopy. 2000, 198(3): 182-187.

[21] J. Ando, G. Bautista, N. Smith, et al., Optical trapping and surgery of living yeast cells using a single laser. The Review of scientific instruments. 2008, 79(10): 103705.

[22] P. Barak, A. Rai, P. Rai, et al., Quantitative optical trapping on single organelles in cell extract. Nature methods. 2013, 10(1): 68-70.

[23] K.C. Neuman, E.H. Chadd, G.F. Liou, et al., Characterization of Photodamage to Escherichia coli in Optical Traps. Biophysical Journal. 1999, 77(5): 28562863.

[24] E.J.G. Peterman, F. Gittes, C.F. Schmidt, Laserinduced heating in optical traps. Biophysical Journal. 2003, 84(2): 1308-1316.

[25] E. Eriksson, J. Enger, B. Nordlander, et al., A microfluidic system in combination with optical tweezers for analyzing rapid and reversible cytological alterations in single cells upon environmental changes. Lab Chip. 2007, 7(1): 7176.

[26] M.P. MacDonald, G.C. Spalding, K. Dholakia, Microfluidic sorting in an optical lattice. Nature. 2003, 426(6965): 421-424.

[27] J.R. Kovac, J. Voldman, Intuitive, image-based cell sorting using optofluidic cell sorting. Analytical chemistry. 2007, 79(24): 9321-9330. 
[28] X. Wang, S. Chen, M. Kong, et al., Enhanced cell sorting and manipulation with combined optical tweezer and microfluidic chip technologies. Lab Chip. 2011, 11(21): 3656-3662.

[29] S.B. Kim, S.Y. Yoon, H.J. Sung, et al., Cross-Type Optical Particle Separation in a Microchannel. Analytical chemistry. 2008, 80(7): 2628-2630.

[30] O. Ostling, K.J. Johanson, Microelectrophoretic study of radiation-induced DNA damages in individual mammalian cells. Biochemical and Biophysical Research Communications. 1984, 123(1): 291-298.

[31] H.A. Pohl, The Motion and Precipitation of Suspensoids in Divergent Electric Fields. Journal of Applied Physics. 1951, 22(7): 869.

[32] F. Kassie, W. Parzefall, S. Knasmuller, Single cell gel electrophoresis assay: a new technique for human biomonitoring studies. Mutat Res-Rev Mutat. 2000, 463(1): 13-31.

[33] T. Tsuda, N. Yamauchi, S. Kitagawa, Separation of red blood cells at the single cell level by capillary zone electrophoresis. Analytical Sciences. 2000, 16(8): 847850.

[34] H.A. Pohl, J.S. Crane, Dielectrophoresis of cells. Biophysical Journal. 1971, 11(9): 711-727.

[35] M. Bocchi, M. Lombardini, A. Faenza, et al., Dielectrophoretic trapping in microwells for manipulation of single cells and small aggregates of particles. Biosensors \& bioelectronics. 2009, 24(5): 1177-1183.

[36] P.R.C. Gascoyne, J. Vykoukal, Particle separation by dielectrophoresis. Electrophoresis. 2002, 23(13): 19731983.

[37] C.C. Wang, K.C. Lan, M.K. Chen, et al., Adjustable trapping position for single cells using voltage phasecontrolled method. Biosensors and Bioelectronics. 2013, 49: 297-304.

[38] C.H. Chuang, Y.W. Huang, Y.T. Wu, Dielectrophoretic chip with multilayer electrodes and micro-cavity array for trapping and programmably releasing single cells. Biomedical microdevices. 2012, 14(2): 271-278.

[39] R.S. Thomas, H. Morgan, N.G. Green, Negative DEP traps for single cell immobilisation. Lab Chip. 2009, 9(11): 1534-1540.

[40] L.S. Jang, P.H. Huang, K.C. Lan, Single-cell trapping utilizing negative dielectrophoretic quadrupole and microwell electrodes. Biosensors \& bioelectronics. 2009, 24(12): 3637-3644.

[41] H. Park, D. Kim, K.S. Yun, Single-cell manipulation on microfluidic chip by dielectrophoretic actuation and impedance detection. Sensors and Actuators B: Chemical. 2010, 150(1): 167-173.

[42] S.H. Kim, T. Yamamoto, D. Fourmy, et al., Electroactive microwell arrays for highly efficient single-cell trapping and analysis. Small. 2011, 7(22): 3239-3247.

[43] C.H. Chuang, Y.W. Huang, Y.T. Wu, System-Level Biochip for Impedance Sensing and Programmable Manipulation of Bladder Cancer Cells. Sensors. 2011, 11(12): 11021-11035

[44] C.H. Chuang, Y.M. Hsu, C.H. Wei, The effects of microstructures on a dielectrophoretic chip for trapping particles. Electrophoresis. 2009, 30(17): 3044-3052.

[45] C.H. Chuang, Y.W. Huang, Y.T. Wu, et al., Programmable Dielectrophoretic Chip for Cell Manipulations. Japanese Journal of Applied Physics. 2011, 50(6): 06GL11.

[46] P.Y. Chiou, A.T. Ohta, M.C. Wu, Massively parallel manipulation of single cells and microparticles using optical images. Nature. 2005, 436(7049): 370-372.

[47] H.Y. Hsu, A.T. Ohta, P.Y. Chiou, et al., Phototransistorbased optoelectronic tweezers for dynamic cell manipulation in cell culture media. Lab Chip. 2010, 10(2): 165-172.
[48] K.W. Huang, Y.C. Wu, J.A. Lee, et al., Microfluidic integrated optoelectronic tweezers for single-cell preparation and analysis. Lab Chip. 2013, 13(18): 37213727.

[49] A.H. Jeorrett, S.L. Neale, D. Massoubre, et al., Optoelectronic tweezers system for single cell manipulation and fluorescence imaging of live immune cells. Optics express. 2014, 22(2): 1372-1380.

[50] N. Pamme, Magnetism and microfluidics. Lab Chip. 2006, 6(1): 24-38.

[51] J. Lipfert, X. Hao, N.H. Dekker, Quantitative modeling and optimization of magnetic tweezers. Biophysical Journal. 2009, 96(12): 5040-5049.

[52] B.M. Lansdorp, S.J. Tabrizi, A. Dittmore, et al., A highspeed magnetic tweezer beyond 10,000 frames per second. The Review of scientific instruments. 2013, 84(4): 044301 .

[53] Y.C. Chung, P.W. Chen, C.M. Fu, et al., Particles sorting in micro-channel system utilizing magnetic tweezers and optical tweezers. Journal of Magnetism and Magnetic Materials. 2013, 333: 87-92.

[54] F.J. Alenghat, B. Fabry, K.Y. Tsai, et al., Analysis of cell mechanics in single vinculin-deficient cells using a magnetic tweezer. Biochem Biophys Res Commun. 2000, 277(1): 93-99.

[55] A.R. Bausch, W. Möller, E. Sackmann., Measurement of Local Viscoelasticity and Forces in Living Cells by Magnetic Tweezers. Biophysical Journal. 1999, 76(1): 573-579.

[56] C. Haber, D. Wirtz, Magnetic tweezers for DNA micromanipulation. Review of Scientific Instruments. 2000, 71(12): 4561-4570.

[57] B. Lim, V. Reddy, X. Hu, et al., Magnetophoretic circuits for digital control of single particles and cells. Nature communications. 2014, 5: 3846.

[58] J. Liu, J. Shi, L. Jiang, et al., Segmented magnetic nanofibers for single cell manipulation. Applied Surface Science. 2012, 258(19): 7530-7535.

[59] H. Ebrahimian, M. Giesguth, K.J. Dietz, et al., Magnetic tweezers for manipulation of magnetic particles in single cells. Appl Phys Lett. 2014, 104(6): 063701.

[60] M.S. Sakar, E.B. Steager, D.H. Kim, et al., Single cell manipulation using ferromagnetic composite microtransporters. Appl Phys Lett. 2010, 96(4): 043705.

[61] S. Miltenyi, W. Muller, W. Weichel, et al., High gradient magnetic cell separation with MACS. Cytometry. 1990, 11(2): 231-238.

[62] K.A. Hyun, T.Y. Lee, S.H. Lee, et al., Two-stage microfluidic chip for selective isolation of circulating tumor cells (CTCs). Biosensors \& bioelectronics. 2014.

[63] J.D. Adams, U. Kim, H.T. Soh, Multitarget magnetic activated cell sorter. Proceedings of the National Academy of Sciences of the United States of America. 2008, 105(47): 18165-18170.

[64] S.C. Lin, X. Mao, T.J. Huang, Surface acoustic wave (SAW) acoustophoresis: now and beyond. Lab Chip. 2012, 12(16): 2766-2770.

[65] M. Gedge, M. Hill, Acoustofluidics 17: theory and applications of surface acoustic wave devices for particle manipulation. Lab Chip. 2012, 12(17): 2998-3007.

[66] X. Ding, P. Li, S.C. Lin, et al., Surface acoustic wave microfluidics. Lab Chip. 2013, 13(18): 3626-3649.

[67] J. Shi, H. Huang, Z. Stratton, et al., Continuous particle separation in a microfluidic channel via standing surface acoustic waves (SSAW). Lab Chip. 2009, 9(23): 33543359.

[68] X. Ding, S.C.S. Lin, B. Kiraly, et al., On-chip manipulation of single microparticles, cells, and organisms using surface acoustic waves. PNAS. 2012, 109(28): 11105-11109. 
[69] J.R. Rettig, A. Folch., Large-scale single-cell trapping and imaging using microwell arrays. Analytical chemistry. 2005, 77(17): 5628-5634.

[70] D. Di Carlo, L.Y. Wu, L.P. Lee. Dynamic single cell culture array. Lab Chip. 2006, 6(11): 1445-1449.

[71] R. Burger, P. Reith, G. Kijanka, et al., Array-based capture, distribution, counting and multiplexed assaying of beads on a centrifugal microfluidic platform. Lab Chip. 2012, 12(7): 1289-1295.

[72] J. Chen, D. Chen, T. Yuan, et al., Microfluidic chips for cells capture using 3-D hydrodynamic structure array. Microsystem Technologies. 2014, 20(3): 485-491.

[73] J.H. Chung, Y.J. Kim, E. Yoon, Highly-efficient singlecell capture in microfluidic array chips using differential hydrodynamic guiding structures. Appl Phys Lett. 2011, 98(12).

[74] J. Fröhlich, H. König, New techniques for isolation of single prokaryotic cells. FEMS Microbiology Reviews.
2000, 24(5): 567-572.

[75] Y. Anis, J. Houkal, M. Holl, et al., Diaphragm picoliter pump for single-cell manipulation. Biomedical microdevices. 2011, 13(4): 651-659.

[76] D.W. Inglis, R. Riehn, J.C. Sturm, et al., Microfluidic high gradient magnetic cell separation. Journal of Applied Physics, 2006, 99(8): 08K101.

[77] G. Paneru, P.S. Thapa, B.N. Flanders, et al., Long reach cantilevers for sub-cellular force measurements. Nanotechnology, 2012, 23(45): 455105.

Copyright $\subseteq 2015$ Shujing Lin, Di Chen, Yao Xie, Binbin Lin, Qian Wei, Fei Pan and Daxiang Cui. This is an open-access article distributed under the terms of the Creative Commons Attribution License, which permits unrestricted use, distribution, and reproduction in any medium, provided the original author and source are credited. 